\title{
Hierro y ácido fólico: natural, enriquecido, fortificado y suplementos. Análisis de las fuentes alimentarias en la Ciudad de Buenos Aires
}

Iron and folic acid: natural, enriched, fortified, and supplements. Analysis of food sources in the Autonomous City of Buenos Aires

\author{
Lic. Mg. María E. Zapata ${ }^{a}$, Bioq. Alicia Rovirosa ${ }^{a}$ y Méd. Esteban Carmuega ${ }^{a}$
}

\section{RESUMEN}

Introducción. La fortificación y suplementación son estrategias para la prevención de carencias de micronutrientes. El objetivo fue describir la procedencia de la ingesta del hierro y ácido fólico a lo largo del ciclo vital de la población de la Ciudad Autónoma de Buenos Aires.

Población y métodos. Análisis de la información dela Primera Encuesta Alimentaria y Nutricional de la Ciudad Autónoma de Buenos Aires 2011, que tomó una muestra probabilística por conglomerados. El consumo se recabó con recordatorio de 24 horas. Se calculó el aporte de hierro y ácido fólico, y se categorizó en contenido natural, harina de trigo enriquecida, leche del Plan Materno Infantil, alimentos fortificados y suplementos.

Resultados. De los 5369 individuos evaluados, prácticamente, la totalidad obtenía hierro y ácido fólico de contenido natural (el $58 \%$ y el $29 \%$ del consumo, respectivamente). Más del $90 \%$ consumía harina de trigo enriquecida, que aportaba el $28 \%$ del hierro y el $54 \%$ del ácido fólico. Los alimentos fortificados mostraron consumo y aporte muy variable. La leche del Plan Materno Infantil mostró muy baja participación, inclusive en grupos específicos. El aporte de suplementos fue bajo, excepto en $<2$ años (el30\% consumía suplementos de hierro, que aportaban el $38 \%$ de este).

Conclusión. Además del aporte natural de los alimentos, la harina de trigo enriquecida representó una importante contribución en el consumo de ácido fólico y hierro de esta población; los alimentos fortificados y los suplementos tuvieron una participación diferente según el grupo etario.

Palabras clave: hierro, ácido fólico, consumo de alimentos, alimentos fortificados, suplementos dietéticos.

http: / / dx.doi.org/10.5546/ aap.2020.160 Texto completo en inglés:

Correspondencia:

Lic. Mg. María E. Zapata: mezapata@cesni.org.ar

Financiamiento:

Ninguno.

Conflicto de intereses: Ninguno que declarar.
Cómo citar: Zapata ME, Rovirosa A, Carmuega E. Hierro y ácido fólico: natural, enriquecido, fortificado y suplementos. Análisis de las fuentes alimentarias en la Ciudad de Buenos Aires. Arch Argent Pediatr 2020; 118(3):160-165.

\section{INTRODUCCIÓN}

La ingesta de nutrientes puede provenir del contenido natural de los alimentos, de la adición de nutrientes en ciertos alimentos ${ }^{1} \mathrm{y}$ del consumo de suplementos. La adición de nutrientes puede establecerse obligatoriamente en casos en que exista una ingesta insuficiente a nivel poblacional con consecuencias negativas para la salud pública (enriquecimiento) o ser fortificación voluntaria, impulsada por la industria alimentaria, ${ }^{2}$ para satisfacer necesidades nutricionales específicas para la población sana y para agregar valor a la venta.

En nuestro país, el Código Alimentario Argentino regula qué alimentos pueden ser fortificados y los niveles de fortificación. ${ }^{3} \mathrm{La}$ fortificación y el enriquecimiento son uno de los principales enfoques para mejorar el consumo de vitaminas y minerales a nivel poblacional, debido a los costos relativamente bajos y los beneficios demostrados, y se consideran como una de las intervenciones de salud pública más rentables para poblaciones con deficiencia de micronutrientes. ${ }^{2}$

La Argentina ha implementado tres estrategias de enriquecimiento de alimentos. En 1967, se dispuso la adición de yodo a la sal para uso humano y animal (Ley 17259). Desde 2001, se estableció la obligatoriedad de adicionar hierro, zinc y vitamina $C$ a la leche entera en polvo que se distribuye a través de programas alimentarios a embarazadas, mujeres en período de lactancia y niños menores de dos años (Ley 25459). En 2002, con el objetivo de prevenir 
la anemia y las malformaciones del tubo neural, se dispuso el enriquecimiento de la harina de trigo con hierro y ácido fólico, y la restitución de tiamina, riboflavina y niacina, que se perdían en la molienda del trigo (Ley 25630), una práctica habitual en muchos países de la región y del mundo. ${ }^{4}$ El suplemento profiláctico se recomienda en los grupos de riesgo. 5,6

La deficiencia de hierro y la anemia son problemas de salud pública universal por sus consecuencias sobre la salud de los individuos y sobre aspectos sociales y/o económicos, que afectan en distinto grado a todos los países. La anemia ocurre a todas las edades, pero su prevalencia es máxima en niños pequeños, mujeres en edad fértil y embarazadas. ${ }^{6}$ Según los datos de Encuesta Nacional de Nutrición y Salud (2005), en la Argentina, el $16 \%$ de los menores de 5 años, el $35 \%$ de los niños de 6 a 24 meses, el $20 \%$ de las mujeres en edad fértil y el $30 \%$ de las embarazas tenía anemia. ${ }^{7}$ La relación entre los defectos del tubo neural y el ácido fólico ha sido reconocida a través de numerosos estudios clínicos y experimentales. En la Argentina, el enriquecimiento de la harina de trigo se asoció con niveles adecuados de folatos en mujeres, tanto de ingesta como séricos. ${ }^{8}$

La información disponible sobre la procedencia de los micronutrientes consumidos en nuestro país es limitada. Este trabajo tiene por objetivo describir el aporte de hierro y ácido fólico de acuerdo con la procedencia de su ingesta en cada etapa del ciclo vital de la población de la Ciudad Autónoma de Buenos Aires (CABA).

\section{POBLACIÓN Y MÉTODOS}

Estudio descriptivo y transversal. Se analizó la información recolectada por la Primera Encuesta Alimentaria Nutricional de la Ciudad de Buenos Aires (EAN-CABA), ${ }^{9}$ realizada, entre mayo y noviembre de 2011, en una muestra probabilística por conglomerados de individuos en diferentes estadios del ciclo vital, que incluyó a niños y niñas de 7 meses a 12 años, adolescentes de ambos sexos, madres con niños $<6$ meses, mujeres en edad fértil y adultos de ambos sexos $>60$ años.

La información del consumo de alimentos y bebidas se recabó mediante un recordatorio de 24 horas, aplicado por nutricionistas entrenados en la técnica. Se consignó el momento de comida, la cantidad y el tipo de alimento o bebida consumido con la especificación de la marca comercial para los productos industrializados. En los $<13$ años, la información fue brindada por las madres o los responsables del cuidado del niño/a. La información acerca del consumo de suplementos de vitaminas y/o minerales se reunió a partir de la indagación sobre el consumo del día previo. Se recolectó la marca comercial, la dosis y el momento de consumo.

Para obtener el contenido de hierro y ácido fólico de alimentos y bebidas, se utilizaron las tablas de composición nutricional de Argenfoods, ${ }^{10}$ del Sistema de Análisis y Registro de Alimentos -software SARA-, ${ }^{11}$ de la base de datos del Departamento de Agricultura de Estados Unidos -United States Department of Agriculture, USDA- ${ }^{12}$ y la información declarada en el rotulado nutricional para los fortificados. El contenido de hierro y ácido fólico de los suplementos se obtuvo de la información provista por cada laboratorio.

Luego fueron categorizados, de acuerdo con la procedencia del hierro y ácido fólico, en los siguientes: 1. Contenido natural (alimentos o bebidas sin adición de hierro o ácido fólico); 2. Harina de trigo (harina de trigo refinada enriquecida, Ley $\mathrm{N}^{\circ} 25630$, y sus subproductos, como, panificados y galletitas); 3 . Leche del Plan Materno Infantil (PMI) enriquecida con hierro, zinc y vitamina $C ; 4$. Fortificados (alimentos y bebidas fortificados con hierro y / o ácido fólico); 5. Suplementos de hierro o ácido fólico.

El análisis estadístico se realizó considerando la ponderación muestral, una técnica estadística que permite corregir desequilibrios en la muestra y obtener datos con mejor representatividad poblacional. Se calcularon medidas de posición, dispersión y porcentajes.

\section{Consideraciones éticas}

Todos los aspectos involucrados en el desarrollo de este proyecto se realizaron adhiriendo a las normativas vigentes nacionales e internacionales. Los adultos participantes y los responsables de los niños y adolescentes firmaron un consentimiento informado.

\section{RESULTADOS}

Se evaluaron 5369 individuos. La Tabla 1 muestra las principales características de cada grupo evaluado. En relación con el hierro, la totalidad de los individuos evaluados consumieron alimentos que naturalmente lo contenían y más del $90 \%$ de los $>2$ años consumió harina de trigo enriquecida o alimentos elaborados (Tabla 2). Los alimentos fortificados en forma voluntaria mostraron una mayor variabilidad; siete de cada diez niños de 
7 a 23 meses incorporaron hierro a partir de estos, básicamente, fórmulas y alimentos infantiles, y uno de cada diez adultos mayores lo hizo. El consumo de leche del PMI era muy bajo, y los suplementos de hierro eran incorporados por un tercio de los niños menores de 2 años, y alrededor del $5 \%$ de los adultos mayores y madres de niños menores de 6 meses.
El contenido natural de los alimentos proveía dos tercios del hierro total y la harina de trigo enriquecida contribuía con un tercio. Los valores más bajos se observaron en los niños de 7 a 23 meses y en los de 2 a 4 años (Tabla 2 y Figura 1). Por su parte, los alimentos fortificados, en promedio, aportaron el $7 \%$ en la población total, y la leche del PMI, el $1 \%$ en los niños

TABLA 1. Características de la muestra $(n=5369)$

\begin{tabular}{|c|c|c|c|c|c|c|c|}
\hline & 7 a 23 meses & $\begin{array}{l}\text { Niños/as } \\
2 \text { a } 4 \text { años }\end{array}$ & 5 a 12 años & $\begin{array}{l}\text { Adolescentes } \\
\text { de } 13 \text { a } 18 \text { años }\end{array}$ & $\begin{array}{l}\text { Madres con niños } \\
\text { < } 6 \text { meses }\end{array}$ & $\begin{array}{l}\text { Mujeres de } \\
19 \text { a } 49 \text { años }\end{array}$ & $\begin{array}{l}\text { Adultos } \\
>60 \text { años }\end{array}$ \\
\hline Muestra (n) & 636 & 765 & 1067 & 920 & 555 & 854 & 572 \\
\hline Muestra ponderada (N) & 62881 & 129369 & 311877 & 212202 & 26786 & 688508 & 629207 \\
\hline Edad (años)* & $1,1 \pm 0,4$ & $2,8 \pm 0,8$ & $8,4 \pm 2,2$ & $15,2 \pm 1,7$ & $29,9 \pm 6,6$ & $33,2 \pm 8,4$ & $71,5 \pm 8,0$ \\
\hline Mujer (\%) & 48,9 & 48,7 & 49,2 & 49,9 & 100 & 100 & 63,1 \\
\hline
\end{tabular}

Nota: * media \pm desvío estándar.

TABLA 2. Consumo de hierro según la procedencia, por etapa del ciclo vital (\% de consumidores y mg/d)

\begin{tabular}{|c|c|c|c|c|c|c|c|c|c|c|c|c|c|c|c|}
\hline & \multicolumn{3}{|c|}{ Natural } & \multicolumn{3}{|c|}{ Harina de trigo } & \multicolumn{3}{|c|}{ Leche del PMI } & \multicolumn{3}{|c|}{ Fortificados } & \multicolumn{3}{|c|}{ Suplementos } \\
\hline & $\%$ & Media & DE & $\%$ & Media & DE & $\%$ & Media & DE & $\%$ & Media & DE & $\%$ & Media & DE \\
\hline Total & 99,9 & 6,6 & 3,8 & 92,4 & 3,2 & 2,6 & 0,5 & 0,0 & 0,4 & 36,7 & 0,8 & 2,3 & 5,5 & 0,8 & 8,3 \\
\hline \multicolumn{16}{|l|}{ Niños/as } \\
\hline 7 a 23 meses & 99,4 & 3,0 & 2,1 & 72,5 & 0,8 & 1,0 & 1,4 & 0,1 & 1,5 & 69,5 & 3,9 & 4,2 & 30,2 & 4,8 & 8,7 \\
\hline 2 a 4 años & 100,0 & 4,5 & 2,7 & 95,2 & 2,2 & 1,8 & 1,0 & 0,1 & 0,8 & 54,4 & 2,1 & 3,3 & 0,8 & 0,0 & 0,2 \\
\hline 5 a 12 años & 99,9 & 5,9 & 3,1 & 96,4 & 3,5 & 2,6 & 0,1 & 0,0 & 0,1 & 45,9 & 1,4 & 2,7 & 0,4 & 0,2 & 3,2 \\
\hline Adolescentes & & & & & & & & & & & & & & & \\
\hline $\begin{array}{l}\text { de13 a } 18 \text { años } \\
\text { Madres con niños }\end{array}$ & 99,9 & 7,2 & 4,2 & 95,2 & 4,7 & 3,6 & 0,1 & 0,0 & 0,4 & 34,0 & 1,2 & 3,0 & 1,4 & 0,1 & 1,6 \\
\hline$<6$ meses & 100,0 & 7,3 & 4,2 & 94,4 & 3,8 & 2,8 & 1,3 & 0,1 & 1,0 & 22,2 & 0,8 & 2,2 & 5,0 & 2,1 & 11,8 \\
\hline Mujeres de 19 a 49 años & 100,0 & 6,9 & 3,6 & 92,4 & 3,1 & 2,5 & 0,0 & 0,0 & 0,0 & 16,2 & 0,5 & 1,7 & 2,9 & 1,2 & 12,5 \\
\hline Adultos $>60$ años & 100,0 & 7,3 & 4,2 & 96,7 & 3,2 & 2,2 & 0,0 & 0,0 & 0,0 & 9,6 & 0,2 & 0,9 & 5,1 & 0,7 & 5,5 \\
\hline
\end{tabular}

DE: desvío estándar; PMI: Plan Materno Infantil.

Figura 1. Aporte de hierro y ácido fólico según la procedencia, por etapa del ciclo vital (\%)

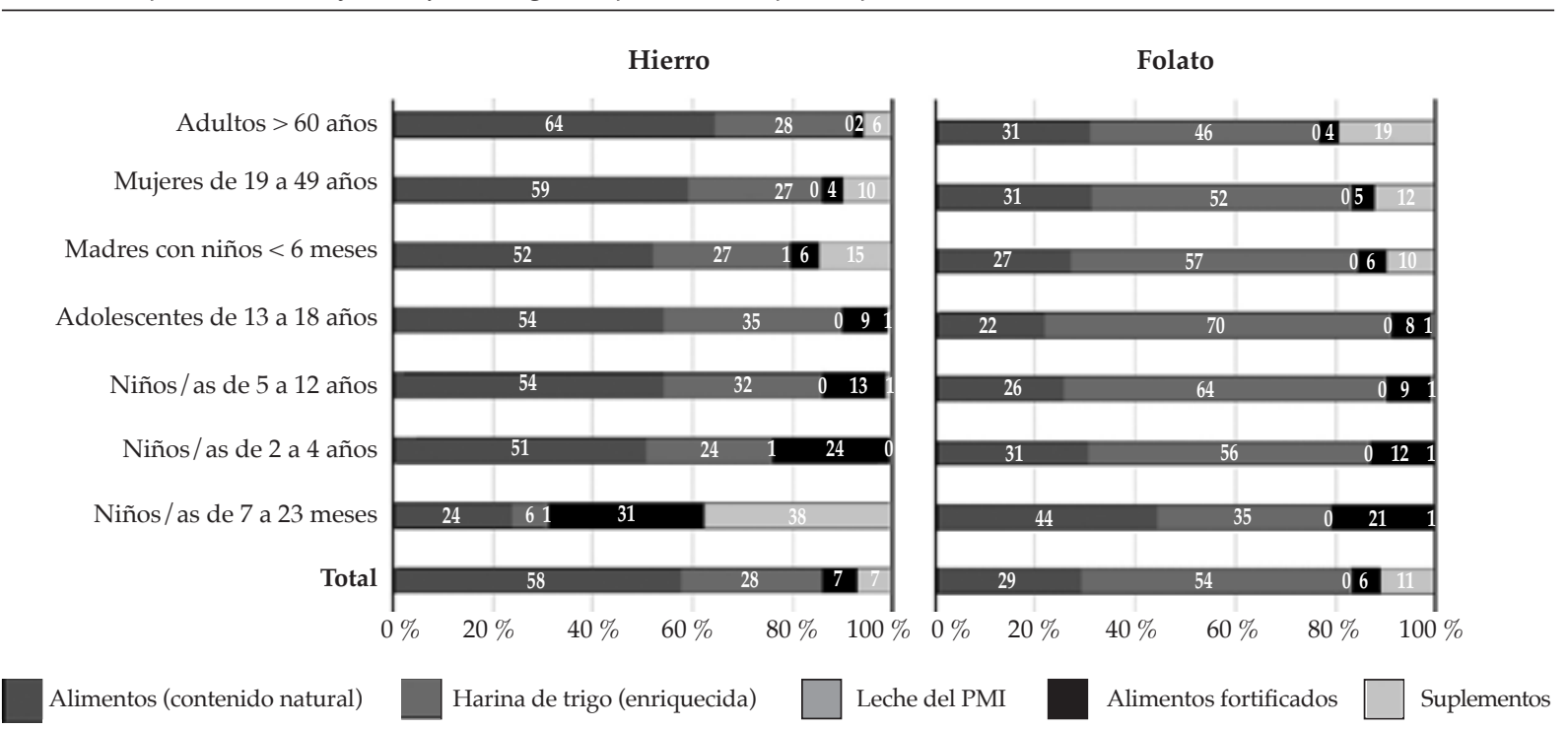

PMI: Plan Materno Infantil. 
menores de 4 años y en las madres de niños de 0 a 6 meses. El aporte de alimentos fortificados se condijo con su mayor o menor consumo a lo largo del ciclo vital; en los niños de 7 a 23 meses, representó un tercio de su ingesta diaria; en los de 2 a 4 años, alcanzó una cuarta parte y, en adultos mayores de 60 años, apenas el $2 \%$ de la ingesta total de hierro provenía de alimentos fortificados (Tabla 2 y Figura 1).

El consumo de folatos de origen natural alcanzaba a más del $99 \%$ de los individuos y su aporte por alimentos fortificados en forma voluntaria era mayor que el observado para el hierro. Cerca de la mitad de la población incorporaba en su alimentación folatos a través de alimentos fortificados, con una frecuencia decreciente a lo largo del ciclo vital. En los menores de 2 años, siete de cada diez los incorporaba y, en los adultos mayores, tres de cada diez. El consumo de suplementos de folatos se concentraba en mujeres adultas y adultos mayores, y, en menor proporción, en los niños y adolescentes (Tabla 3).

La harina de trigo enriquecida era la principal fuente de folato de la alimentación de la población evaluada. Contribuía con más de la mitad, seguida por los alimentos que lo contenían en forma natural y luego los fortificados en forma voluntaria. El aporte de la harina de trigo oscilaba entre el $35 \%$ y el $70 \%$ en niños de 7 a 23 meses y adolescentes, respectivamente. El ácido fólico de origen natural mostraba el valor más bajo en los adolescentes (el $22 \%$ ) y el más alto en los niños de 7 a 23 meses (el $44 \%$ ). El aporte de los alimentos fortificados en forma voluntaria disminuía a medida que aumentaba la edad: en los niños de 7 a 23 meses, representaba el $21 \%$; el $12 \%$ en los de 2 a 4 años y menos del $10 \%$ en el resto de los grupos. Los suplementos aportaban el $19 \%$ del total consumido por los adultos mayores y más del $10 \%$ en las madres y mujeres en edad fértil, mientras que, por el bajo consumo de suplementos en las otras etapas de la vida, el aporte de los suplementos era mínimo (Tabla 3 y Figura 1).

\section{DISCUSIÓN}

La carencia de micronutrientes es un tema de creciente interés, ${ }^{13}$ debido a que contribuye, de manera sustancial, a la carga mundial de morbilidad y porque, además, es un problema de salud pública que afecta tanto a países desarrollados como en vías de desarrollo y a las poblaciones vulnerables en mayor medida. ${ }^{4}$ Una dieta equilibrada que sea adecuada en cuanto a todos los nutrientes es la mejor manera para prevenir carencias, pero, para ello, es necesario el acceso universal a alimentos adecuados y hábitos de alimentación apropiados.

Es por esto por lo que se han implementado estrategias para prevenir carencias de aquellos nutrientes que suponen problemas de salud pública, como yodo, vitamina $\mathrm{A}$, hierro y ácido fólico, entre otros. La fortificación de alimentos, tanto obligatoria (enriquecimiento) como voluntaria (fortificación), masiva o focalizada, es considerada una intervención de salud pública costo-efectiva para el control de la carencia de micronutrientes y tiene la ventaja doble de proporcionar nutrientes a grandes segmentos de la población sin necesitar de cambios radicales en los patrones de consumo de alimentos. El suplemento específico a grupos de riesgo o vulnerables mejora más rápidamente el estado nutricional. ${ }^{4}$ No se han encontrado datos locales disponibles sobre la procedencia de consumo de hierro y ácido fólico.

TABLA 3. Consumo de ácido fólico según la procedencia, por etapa del ciclo vital (\% de consumidores y mg/d)

\begin{tabular}{|c|c|c|c|c|c|c|c|c|c|c|c|c|}
\hline & \multicolumn{3}{|c|}{ Natural } & \multicolumn{3}{|c|}{ Harina de trigo } & \multicolumn{3}{|c|}{ Fortificados } & \multicolumn{3}{|c|}{ Suplementos } \\
\hline & $\%$ & Media & DE & $\%$ & Media & DE & $\%$ & Media & DE & $\%$ & Media & DE \\
\hline Total & 99,8 & 130 & 115 & 92,4 & 239 & 234 & 46,2 & 26 & 51 & 1,9 & 49 & 612 \\
\hline \multicolumn{13}{|l|}{ Niños/as } \\
\hline 7 a 23 meses & 99,2 & 79 & 60 & 72,5 & 62 & 89 & 70,9 & 37 & 43 & 0,0 & 0 & 0 \\
\hline 2 a 4 años & 99,9 & 87 & 58 & 95,2 & 160 & 164 & 60,3 & 36 & 49 & 0,1 & 1 & 34 \\
\hline 5 a 12 años & 99,9 & 105 & 85 & 96,4 & 264 & 239 & 56,4 & 36 & 51 & 0,3 & 4 & 140 \\
\hline Adolescentes de 13 a 18 años & 99,9 & 114 & 96 & 95,2 & 361 & 324 & 44,2 & 41 & 76 & 1,4 & 3 & 41 \\
\hline Madres con niños $<6$ meses & 100,0 & 136 & 128 & 94,4 & 288 & 233 & 32,1 & 28 & 60 & 4,9 & 49 & 431 \\
\hline Mujeres de 19 a 49 años & 99,9 & 135 & 116 & 92,4 & 226 & 222 & 27,3 & 21 & 46 & 3,4 & 53 & 676 \\
\hline Adultos $>60$ años & 99,8 & 157 & 134 & 96,7 & 232 & 206 & 26,6 & 20 & 43 & 5,3 & 97 & 838 \\
\hline
\end{tabular}

DE: desvío estándar. 
Los resultados muestran la importante contribución al consumo de hierro y ácido fólico de los alimentos enriquecidos y fortificados, especialmente, el ácido fólico de la harina de trigo, que duplica el aporte natural de los alimentos, y el hierro de fórmulas y alimentos infantiles fortificados, que contribuye, en gran medida, a la ingesta en los niños. El aporte de los suplementos es significativo en grupos específicos, como el hierro en los $<2$ años, para los cuales existen directrices que así lo indican. ${ }^{6}$ Por las características de la dieta argentina, el hierro natural de los alimentos, que contribuye con más de la mitad del consumo, mayormente, proviene de carnes, por lo que se considera una mayor biodisponibilidad, ${ }^{14}$ en relación con el proveniente de otros alimentos que lo contienen en forma natural, como vegetales y legumbres, y del incorporado mediante la fortificación o el enriquecimiento. La harina de trigo es enriquecida con sulfato ferroso, que es un compuesto de buena biodisponibilidad. ${ }^{4}$

El enriquecimiento de harina de trigo con hierro es una norma en 84 países y, con ácido fólico, en 80 países, ${ }^{15} \mathrm{y}$ la evidencia ha demostrado que el enriquecimiento de la harina de trigo con hierro reduce la anemia por deficiencia de hierro ${ }^{16}$ $\mathrm{y}$, con ácido fólico, disminuye los defectos del tubo neural, deficiencia de folato y anemia por deficiencia de folato. ${ }^{17} \mathrm{El}$ consumo de ácido fólico a partir de harina de trigo y alimentos elaborados con ella es la principal fuente en la dieta en todos los grupos etarios, a excepción de los niños de 7 a 23 meses.

Estos datos concuerdan con los observados en el análisis realizado a partir de las encuestas de gastos de hogares, ${ }^{14}$ que demostró que más de dos terceras partes de la ingesta aparente de folatos de la población argentina provenía de harina, panificados y galletitas, y que el consumo de ácido fólico se había cuadriplicado luego de la implementación de la ley, y la harina y los derivados eran los principales responsables del aumento (de 64 ug en 1996-97 a 588 ug en 2012-13, por día por adulto equivalente). Además, a nivel nacional, el enriquecimiento de la harina de trigo con ácido fólico se asoció con ingestas y niveles séricos adecuados de folatos en las mujeres, y se observó una reducción significativa en la prevalencia y mortalidad de los defectos del tubo neural. ${ }^{8}$

En el caso del hierro, la harina de trigo tiene una contribución importante pero menor que los folatos. Del análisis de la Encuesta Nacional de Gastos de Hogares ${ }^{14}$ se desprende que los productos elaborados con harina aportan cerca de la mitad (el $44 \%$ ) del hierro de la dieta de la población argentina, con un aumento entre la primera y la última encuesta como consecuencia de la ley, en la que la harina de trigo y los derivados pasaron de aportar $3,5 \mathrm{mg}$ en 1996-97 a 7,0 mg en 2012-13 por día por adulto equivalente. Los alimentos fortificados en forma voluntaria representan una opción cada vez más frecuente en el mercado, especialmente, aquellos orientados a grupos poblacionales específicos. En los resultados, se observa que, si bien están presentes en todos los grupos etarios evaluados, su presencia decrece a medida que aumenta la edad.

Una de las principales fortalezas de este trabajo es el uso de una gran muestra representativa de cada etapa del ciclo vital de una de las principales ciudades del país. Entre las limitaciones, es necesario considerar que se utilizó un recordatorio de 24 horas como fuente de obtención de la información de consumo, que es un buen método para estimar la ingesta media, pero puede subestimar el porcentaje de consumidores y, cuando solo se realiza uno, no es suficiente para calcular el riesgo de ingesta inadecuada. Otra limitación para considerar es la dificultad de diferenciar el contenido natural de hierro y ácido fólico en los alimentos fortificados y enriquecidos, por lo cual se consideró el contenido total como el total de cada categoría, lo que, posiblemente, sobrestimó el aporte de ambas categorías.

Contar con información sobre las fuentes que proveen hierro y ácido fólico en la dieta a lo largo del ciclo vital es muy importante en términos de evaluar el impacto poblacional de las políticas de enriquecimiento, el aporte natural de los alimentos y el consumo de alimentos fortificados. Para complementar y ampliar esta información, sería de gran interés evaluar el riesgo de ingesta inadecuada, tanto insuficiente como excesiva, la cobertura de recomendaciones, como así también el estado nutricional con respecto a estos nutrientes, especialmente, en los grupos poblacionales de riesgo. Los datos de este trabajo demuestran que, además del aporte natural de los alimentos, la harina de trigo enriquecida representa una importante contribución en el consumo de ácido fólico y hierro de toda la población, mientras que los alimentos fortificados y los suplementos tienen una participación diferente según el grupo etario. 


\section{REFERENCIAS}

1. Fulgoni VL 3rd, Keast DR, Bailey RL, Dwyer J. Foods, fortificants, and supplements: Where do Americans get their nutrients? J Nutr. 2011; 141(10):1847-54.

2. World Health Organization. WHO Meeting on estimating appropriate levels of vitamins and minerals for food fortification programmes: The WHO Intake Monitoring, Assessment and Planning Program (IMAPP): meeting report. Geneva: WHO: 2010.

3. Ministerio de Salud de la Nación. Alimentos de régimen o dietéticos. Artículo 1347 - (Res 1542, 12.09.90). En Código Alimentario Argentino. 2013. [Acceso: 22 de octubre de 2019]. Disponible en: http:/ /www.alimentosargentinos. gob.ar/contenido/marco/CAA/Capitulo_17.htm.

4. Allen L, De Benoist B, Dary O, Hurrell R (eds.). Guidelines on food fortification with micronutrients. París: World Health Organization, Food and Agricultural Organization of the United Nations; 2006.

5. Abeyá Gilardon E, Calvo E, Durán P, Longo E, et al. Evaluación del estado nutricional de niñas, niños y embarazadas mediante antropometría. Buenos Aires: Ministerio de Salud de la Nación; 2009.

6. Comité Nacional de Hematología, Oncología y Medicina Transfusional, Comité Nacional de Nutricion. Deficiencia de hierro y anemia ferropenica. Guia para su prevencion, diagnostico y tratamiento. Resumen ejecutivo. Arch Argent Pediatr. 2017; 115(4):406-8.

7. Ministerio de Salud de la Nación. Encuesta Nacional de Nutrición y Salud. Documento de resultados. Buenos Aires: MINSAL; 2007.

8. Calvo E, Biglieri A. Impacto de la fortificación con ácido fólico sobre el estado nutricional en mujeres y la prevalencia de defectos del tubo neural. Arch Argent Pediatr. 2008; 106(6):492-8.

9. Gobierno de la Ciudad Autónoma de Buenos Aires. Primera Encuesta Alimentaria Nutricional de la Ciudad de Buenos Aires. Documento de resultados 2011. Buenos Aires; MINSAL; 2013.

10. Argenfoods. Tablas de composición de alimentos. Universidad de Luján; 2016. [Acceso: 22 de octubre de 2019]. Disonible en: http://www.argenfoods.unlu.edu. ar/Tablas/Tabla.htm.

11. Ministerio de Salud de la Nación, Dirección Nacional de Salud Materno Infantil.SoftwareSARA. Sistema de Análisis y Registro de Alimentos, Versión 1.2.22 ed. 2007.

12. US Departament of Agriculture, Agricultural Research Service. Food Data Central. [Acceso: 22 de octubre de 2019]. Disonible en: https: / / fdc.nal.usda.gov/.

13. Mozaffarian D, Rosenberg I, Uauy R. History of modern nutrition science-implications for current research, dietary guidelines, and food policy. BMJ. 2018; 361:k2392.

14. Zapata ME, Rovirosa A, Carmuega E. La mesa argentina en las últimas dos décadas. Cambios en el patrón de consumo de alimentos y nutrientes (1996-2013). Buenos Aires: CESNI; 2016.

15. Pachón H. History of Food Fortification. New Delhi: Food Fortification iniciative; 2016.

16. Pachón H, Spohrer R, Mei Z, Serdula MK. Evidence of the effectiveness of flour fortification programs on iron status and anemia: a systematic review. Nutr Rev. 2015; 73(11):780-95.

17. Arth A, Kancherla V, Pachón H, Zimmerman S, et al. A 2015 global update on folic acid-preventable spina bifida and anencephaly. Birth Defects Res A Clin Mol Teratol. 2016; 106(7):520-9. 